\title{
Histoplasmose buccale révélatrice d'un syndrome d'immunodéficience acquise. À propos d'un cas.
}

\section{Oral histoplasmosis revealing an acquired immunodeficiency syndrome. A case report.}

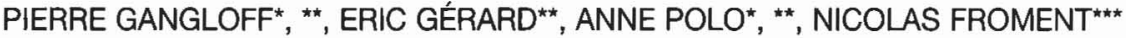

\section{RÉSUMÉ}

L'histoplasmose est une mycose granulomateuse intracellulaire intéressant principalement le système réticulo-endothélial. Deux formes humaines ont été décrites selon l'agent infectieux en cause : Histoplasma capsulatum pour la forme américaine et Histoplasma duboisii pour la forme africaine. En Europe, seuls quelques cas isolés ont été signalés. Quarante pour cent des patients infectés présentent des ulcérations de la muqueuse buccale. Le diagnostic repose sur les examens histologiques et mycologiques. Cette infection représente la troisième mycose opportuniste majeure observée dans l'infection au VIH, elle constitue un critère de passage au stade de SIDA. Le plan de traitement repose d'abord sur l'utilisation d'amphotéricine $B$ jusqu'à guérison des lésions puis sur l'itraconazole jusqu'à récupération par le patient d'une immunité correcte. Ce diagnostic doit être évoqué devant l'augmentation de la mobilité des populations. (Med Buccale Chir Buccale 2002; 8: 91-5).

mots clés : Mycoses, Histoplasmose, Infection à VIH, Muqueuse buccale.

médecine buccale chirurgie buccale

VOL. $8, \mathrm{~N}^{\circ} 2$ 2002

page 91

\section{SUMMARY}

Histoplasmosis is an intracellular granulomatosis mycosis of the reticulo-endothelial system. Two human types have been described according to infectious agent involved: Histoplasma capsulatum for American type and Histoplasma duboisii for African type. This systemic mycosis has the capacity to disseminate from the lung to the oral mucosa. In Europe, only few cases have been reported. Forty percent of infected patients present oral mucosa ulcerations, which are always painful. The diagnostic is obtained by histological, with specific coloration of Gomori-Grocott, and mycological examinations. This infection represents the third opportunistic mycosis that affects the HIV positive patient and indicates the change to AIDS state. The treatment plan involves the use of amphotericin $B$ for the lesions remission, and, following this, itraconazole is administered until patient recovers an acceptable immunity. The diagnostic has to be evoked due to the increase of population's mobility. (Med Buccale Chir Buccale 2002; 8: 91-5).

key words : Mycoses, Histoplasmosis, HIV infection, Oral mucosa.

\footnotetext{
* Département de Pathologie et de Chirurgie Buccales - CHRU de Nancy, France

** Service d'Odontologie, CHR de Metz - Thionville, France

*** Service d'Anatomie et de Cytologie Pathologiques, CHR de Metz - Thionville, France

Demande de tirés à part :

Pierre Gangloff Départernent de Pathologie et de Chirurgie Buccales Faculté de Chirurgie Dentaire 96, avenue de Lattre de Tassigny BP 5020854004 Nancy Cedex
} 
L'histoplasmose est une mycose granulomateuse systémique intra-cellulaire du système réticulo-endothélial. Elle est caractérisée par un syndrome général, associé ou non à des ulcérations de la muqueuse buccale [1].

On distingue deux histoplasmoses humaines:

- l'histoplasmose classique américaine, dite à petites formes, due à Histoplasma capsulatum, décrite initialement par Darling [2] ; forme rapportée dans le cas clinique présenté ;

- I'histoplasmose africaine, dite à grandes formes, due à Histoplasma duboisii.

Cette pathologie représente une infection opportuniste du SIDA [3], fréquente aux EtatsUnis et en Afrique mais exceptionnelle en France.

\section{OBSERVATION}

médecine buccale chirurgie buccale

VOL. $8, N^{\circ} 2$ 2002

page 92
Une femme de 30 ans, originaire du Vietnam, consulte pour une ulcération douloureuse de la gencive. L'examen endobuccal révèle une lésion ulcéro-bourgeonnante de la muqueuse gingivale en regard du bloc incisivo-canin inférieur intéressant aussi bien le versant vestibulaire que lingual. Les bords sont infiltrés et le fond bourgeonnant, donnant à la lésion un aspect pseudo-néoplasique (Fig. 1). Les dents ne présentent pas de mobilité anormale. L'examen exobuccal révèle des adénopathies sous-maxillaires bilatérales. L'examen clinique général est sans particularité. Devant l'aspect suspect mais non spécifique de la lésion, une biopsie est confiée au service d'anatomie et de cytologie pathologiques. L'examen histologique montre alors une infiltration du chorion par une prolifération tumorale, agencée en nappes ou plages massives composées de cellules de taille moyenne avec un cytoplasme granuleux, éosinophile et un noyau arrondi ou ovalaire, finement nucléolé. La muqueuse sus-jacente présente une hyperplasie pseudo-épithéliomateuse (Fig. 2). Le diagnostic retenu par l'histologiste est celui de tumeur à cellules granuleuses d'Abrikossoff. L'absence de corrélation entre l'examen clinique et le diagnostic proposé fait pratiquer des investigations complémentaires qui révèlent une sérologie positive pour le virus de l'immunodéficience humaine. Une deuxième biopsie de la lésion est alors réalisée et envoyée en informant l'histologiste sur le statut sérologique de la patiente. Sur ce nouveau prélèvement, il y a une population de cellules d'allure histiocytaire renfermant de multiples petites granulations cernées par un petit halo clair et infiltrant le chorion. Après une coloration par le Giemsa lent (coloration de May-GrünwaldGiemsa modifié) et sous fort grossissement, une zone claire périphérique, peu colorée, biréfringente est observée, réalisant une image de pseudocapsule (Fig. 3). Avec la coloration de Gomori-Grocott, les histoplasmes intracellulaires, correspondant aux particules noires de 3 microns de diamètre, sont bien visibles (Fig. 4). Cette biopsie, associée à une mise en culture, a donc permis d'infirmer le diagnostic initial et de

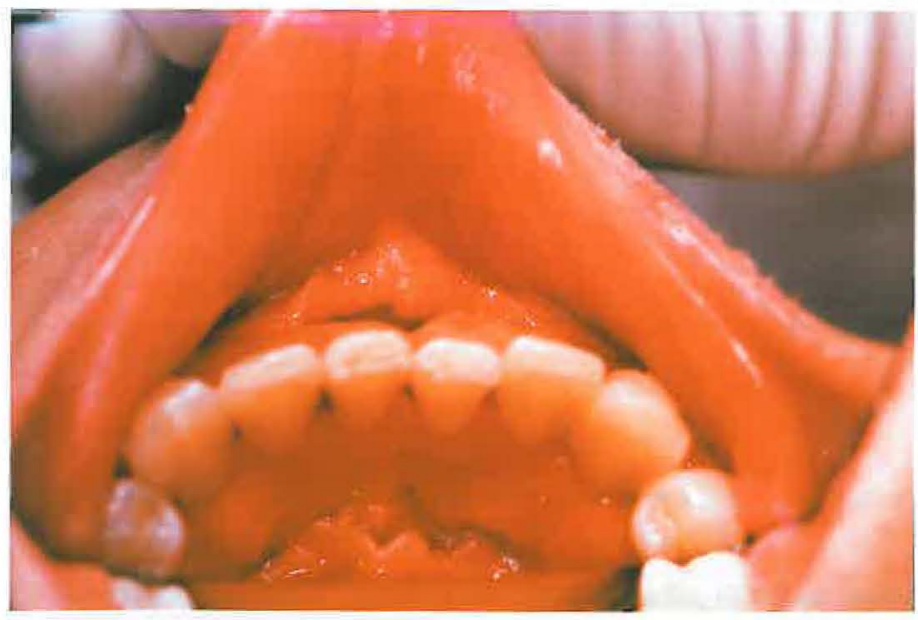

Figure 1 : Lésion ulcéro-bourgeonnante, pseudo-néoplasique intéressant la gencive vestibulaire et linguale dans la région incisivo-canine mandibulaire (aspect lors de la première consultation).

Ulcerated, granulating lesion, pseudoneoplasic appearance of vestibular and buccal gum in incisal-canine area (first consultation). 

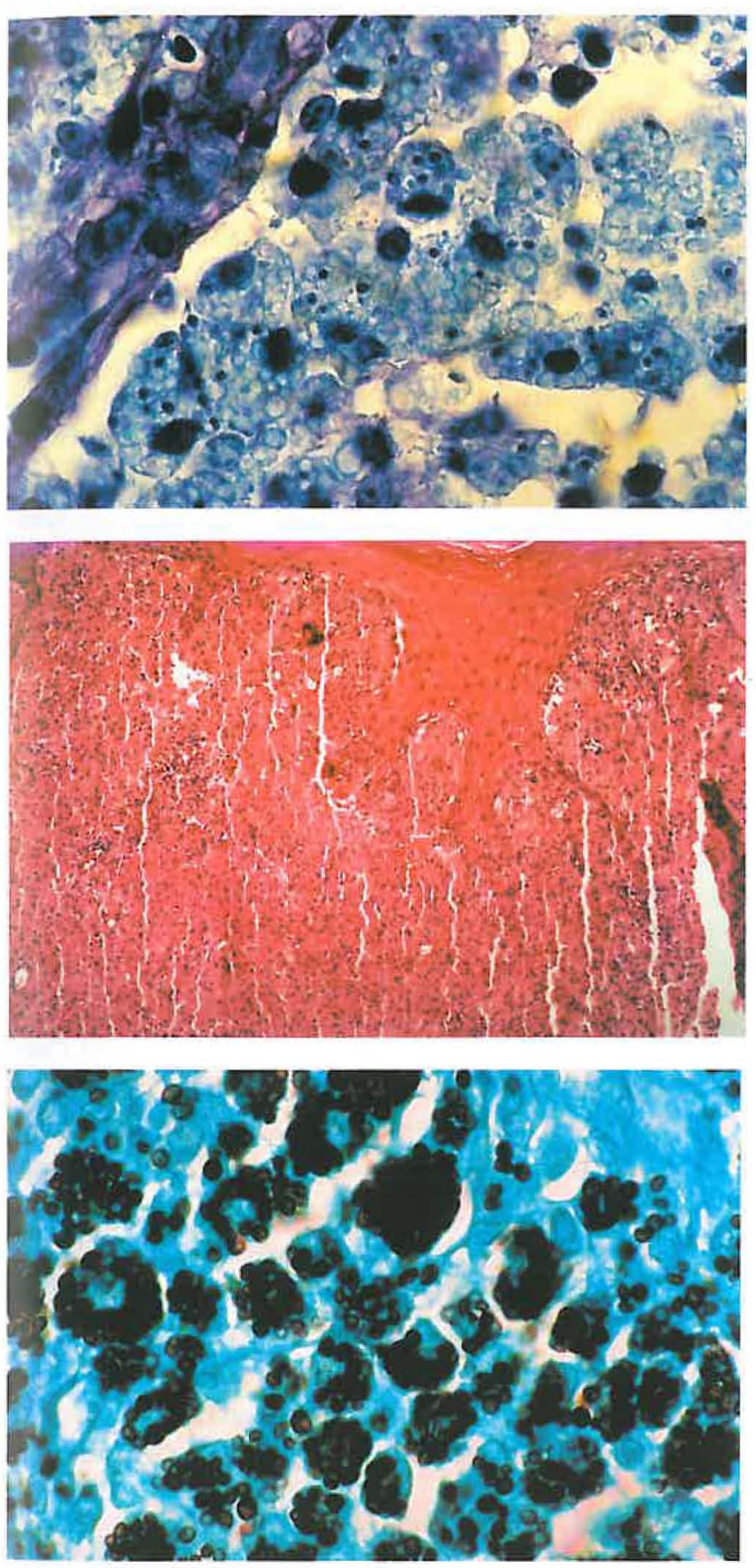

Figure 2 : Coupe histologique de la première biopsie. Coloration de May-Grünwald-Giemsa. Grossissement $\times 6$. L'épithélium repose sur un infiltrat dense composé d'histiocytes à cytoplasme éosinophile. A ce grossissement, on n'observe pas de cellules granuleuses.

Histological section from first biopsy. MayGrünwald-Giemsa coloration. Original magnification $x 6$. The epithelium is lying above an infiltrat made of hystiocyts with eosinophil cytoplasm. At this magnification, no granulatosis cells are observed.

Figure 3 : Coupe histologique de la deuxième biopsie. Coloration de Giemsa lent. Grossissement x 100. Une zone claire, périphérique, biréfringente réalisant une pseudo-capsule est observée.

Histological section from second biopsy. Giemsa slow coloration. Original magnification $\times 100$. A clear zone, peripheric, birefringent leading to a pseudo-capsule is observed.

Figure 4 : Coupe histologique de la deuxième biopsie. Coloration de Gomori-Grocott. Grossissement $\times 100$. Les histoplasmes intracellulaires sont observés sous forme de particules noires.

Histological section from second biopsy. Gomori Grocott coloration. Original magnification x 100. Intra-cellular histoplasms are observed as black particles. médecine

buccale

chirurgie

buccale

VOL. $8, \mathrm{~N}^{\circ} 2$ 2002

page 93 conclure à une histoplasmose de la muqueuse gingivale. La patiente est alors adressée au service des maladies infectieuses. Un bilan d'extension est pratiqué étant donné la possibilité de localisations multiples, essentiellement pulmonaires. La radiographie pulmonaire, la fibroscopie bronchique ainsi que la culture du lavage alvéolaire sont sans particularité. Le traitement a consisté en l'administration d'amphotéricine B (2 mg/kg) par voie intra-veineuse lente, un jour sur deux. Ce traitement a été maintenu au-delà de la cicatrisation de la lésion gingivale puis relayé par de l'itraconazole (200 mg/j) jusqu'à ce que la patiente retrouve une immunité suffisante. Le suivi est régulier afin de détecter précocement toute éventuelle récidive. 


\section{COMMENTAIRES}

L'infection par Histoplasma capsulatum se transmet par inhalation des spores dans les régions contaminées, principalement le Sud-Est, le centre et la Côte Est des Etats-Unis et les Antilles françaises. Dans ces régions, le test à l'histoplasmine réalisé par une intradermo-réaction se révèle positif pour $80 \%$ de la population [4]. II n'y a pas de contamination interhumaine.

Histoplasma capsulatum est un champignon dimorphique : il se présente sous forme de levure dans les tissus et en culture à $37^{\circ} \mathrm{C}$ (forme parasitaire) et sous forme filamenteuse en culture à $30^{\circ} \mathrm{C}$ (forme mycélienne) [5]. L'agent infectieux, après inhalation, va parasiter le système réticulo-endothélial. De multiples granulations se développent au sein du cytoplasme des histiocytes et des cellules multinucléées. Elles représentent la forme parasitaire de l'agent infectieux et ont un diamètre compris entre 2 et 5 microns. Pour les lésions cutanéomuqueuses, le diagnostic positif repose principalement sur l'examen histologique après biopsie [6] et requiert une coloration spéciale, la meilleure étant la coloration de Gomori-Grocott. Une culture peut être pratiquée également afin de mettre en évidence les colonies d'Histoplasma capsulatum. L'examen histologique montre de nombreuses spores PAS positives au sein du cytoplasme avec une paroi biréfringente. De nombreux histiocytes sont également retrouvés.

L'histoplasmose peut évoluer sous deux formes: la forme aiguë et la forme chronique. La forme aiguë, ou primo-infection, peut se limiter à une atteinte pulmonaire asymptomatique $(95 \%$ des cas) ou peut se révéler disséminée d'emblée (5\% des cas). La forme chronique comporte deux tableaux cliniques: la forme chronique pulmonaire et la forme chronique disséminée. Cette dissémination peut être viscérale, et se révéler alors le plus souvent mortelle, ou buccale provoquant des lésions muqueuses dans $30 \%$ des cas qui prennent alors une valeur diagnostique [7]. La forme chronique disséminée s'observe rarement chez des patients sains. Sa fréquence augmente chez les enfants, les personnes âgées et les patients immunodépri- més [7]. Sa découverte doit alerter le praticien et faire rechercher une infection à VIH. L'infection chronique disséminée représente aujourd'hui la troisième mycose opportuniste chez les sujets atteints du SIDA [5]. L'histoplasmose constitue la première mycose d'importation en France [5].

La topographie préférentielle des lésions buccales est, par ordre de fréquence décroissante, la gencive $(33 \%)$, la langue $(25 \%)$, le palais $(21 \%)$ et l'oropharynx (5\%) [8]. Les manifestations buccales n'étant pas spécifiques [9, 10], le diagnostic différentiel peut faire discuter un carcinome épidermoïde, une tuberculose, un lymphome ou une granulomatose de Wegener [11]. L'amphotéricine $\mathrm{B}$ par voie veineuse à la posologie de $2 \mathrm{mg}$ par jour $[7,9]$ représente le traitement recommandé de l'histoplasmose [12]. Elle agit au niveau de la membrane cellulaire, inhibe l'activité métabolique et provoque une perte du contenu intracellulaire [13]. Une fois la cicatrisation de la lésion muqueuse obtenue, l'amphotéricine $B$ est relayée par de l'itraconazole per os à la posologie de $200 \mathrm{mg} / \mathrm{j}$ pendant 6 mois [14]. Dans les cas d'intolérance à l'amphotéricine $\mathrm{B}$, l'itraconazole peut être utilisé seul par voie intraveineuse [15]. Contrairement à l'infection à Histoplasma capsulatum, l'infection à Histoplasma duboisii comporte rarement des atteintes muqueuses. Cette dernière se rencontre en Afrique noire et à Madagascar. L'agent infectieux n'a pas été isolé dans la nature et son mode de transmission chez l'homme est encore incertain [5]. Une porte d'entrée cutanée semble probable. La plupart des observations pourraient correspondre à une contamination digestive abdominale [4]. Le traitement repose également sur l'amphotéricine B et l'itraconazole.

L'histoplasmose reste une maladie exceptionnelle en Europe (69 cas publiés en 14 ans) [16] mais il faut savoir y penser chez les patients ayant séjourné dans les zones infectées ou chez les patients séro-positifs pour le VIH. La dissémination de l'histoplasmose peut intéresser la muqueuse buccale, soit comme signe révélateur de la maladie soit comme signe d'accompagnement.

Devant l'aspect clinique fortement évocateur d'une lésion maligne, le diagnostic positif 
repose principalement sur les examens anatomo-pathologiques et mycologiques.

Devant un diagnostic avéré d'histoplasmose, il faut évoquer la possibilité que le patient soit infecté par le virus VIH [17, 18]. En effet, l'histoplasmose représente la troisième mycose majeure opportuniste dans ce cas. Chez ces patients infectés par le virus de l'immunodéficience humaine, la découverte d'une histoplasmose marque le passage au stade SIDA et en aggrave fortement son diagnostic [1].

\section{RÉFÉRENCES}

1. Fraysse E, Grunenwald J, Dubertrand Y, Flach FX, BARNIER G. Histoplasmose buccale. Rev Stomatol Chir Maxillofac 1990; 91: 128-30.

2 - DARLING ST. A protozoan general infection producing pseudo-tubercles in the lungs and focal necrosis in the liver, spleen and lymph nodes. JAMA 1906; 46: $1283-5$.

3 - SWindells S, DuRHam T, Johansson SL, Kaufman L. Oral histoplasmosis in a patient infected with HIV. A case report. Oral Surg Oral Med Oral Pathol 1994; 77: 126-30.

4 - Kuffer R, Badillet G. Mycoses buccofaciales. Encycl Méd Chir, Elsevier, Paris, Stomatol-Odontol 1995, 22-045-M-10, $30 \mathrm{p}$.

5 - Cremer G. Manifestations cutanées des mycoses profondes et exotiques. Encycl Méd Chir Elsevier, Paris, Dermatol 2000, 98-390-A-10, $12 \mathrm{p}$.

6 - Loubière $R$, Tereau $Y$, Grunenwald J, Marty P, Saint PAUL MC. Buccal histoplasmosis. A propos of a case with a difficult histopathological diagnosis. Ann Pathol 1988; 8: 328-31.

7- Eisig S, Boguslaw B, Cooperband B, Phelan J. Oral manifestations of disseminated histoplasmosis in acquired immunodefiency syndrome: report of two cases and review of the literature. $J$ Oral Maxillofac Surg 1991; 49: 310-3.

8 - HAN NG, HUAT SIAR C. Review of oral histoplasmosis in Malaysia. Oral Surg Oral Med Oral Pathol 1996; 81: 303-7.

9 - OdA D, MCDOUgal L, FRITSChe T, WORTHINGTON P. Oral histoplasmosis as a presenting disease in acquired immunodefiency syndrome. Oral Surg Oral Med Oral Pathol 1990; 70: 631-6.

10 - Gomes ferreira O, Vieira fernandes A, Sebastiao Borges A, Simao Ferreira M, Mota Loyola A. Orofacial manifestations of histoplasmosis in HIV-positive patients: a case report. Med Oral 2001; 6: 101-5.
11 - LASKAPIS G. Infections fongiques. In: Atlas des maladies buccales. Laskaris G. Ed : Flammarion, Paris 1994.

12 - MEYeR RD. Cutaneous and mucosal manifestations of the deep mycotic infections. Acta Derm Venereol Suppl (Stockh) 1986; 121: 57-72.

13 - Sande MA, MandeLL GL. Antimicrobial agents (pp. 1232-1236). In: The pharmacological basis of therapeutics. AG Gilman, LS Goodman, A Gilman. Ed : McMillan, New York 1980.

14 - Clyti E, Aznar C, Couppie P, Sainte-Marie D, Lemoine C, Huerre M, El Guedu M, Dupont B, Cafme B, Pradinaud R. Histoplasmose disséminée révélée par des érosions linguales et amygdaliennes chez un malade immunocompétent. Ann Dermatol Venereol 1999; 126: 709-11.

15 - SLain D, Rogers PD, CLeARY JD, Chapman SW. Intraveinous itraconazole. Ann Pharmacother 2001; 35: $720-9$.

16 - Manfredi R, Mazzoni A, Nanetti A, Chiodo F. Histoplasmosis capsulati and duboisii in Europe: the impact of the HIV pandemic, travel and immigration. Eur J Epidemiol 1994; 10: 675-81.

17 - JING W, ISMAIL R. Mucocutaneous manifestations of HIV infection: a retrospective analysis of 145 cases in a Chinese population in Malaysia. Int $J$ Dermatol 1999; 38: 457-63.

18 - Casariego Z, Kelly gr, Perez $\mathrm{H}$, Cahn P, Guelfan L, Kaufman S, Scully C. Disseminated histoplasmosis with orofacial involvement in HIV-1-infected patients with AIDS: manifestations and treatment. Oral Dis 1997; 3: 184-7. médecine

buccale

chirurgie

buccale

VOL. $8, N^{\circ} 2$ 2002

page 95 